\title{
Doppler ultrasound in liver cirrhosis: correlation of hepatic artery and portal vein measurements with model for end-stage liver disease score in Egypt
}

Ahmed Abdelrahman Mohamed Baz ${ }^{1 *}$, Rana Magdy Mohamed ${ }^{2}$ and Khaled Helmy El-kaffas ${ }^{1}$

\begin{abstract}
Background: Liver cirrhosis is a multi-etiological entity that alters the hepatic functions and vascularity by varying grades. Hereby, a cross-sectional study enrolling 100 cirrhotic patients (51 males and 49 females), who were diagnosed clinically and assessed by model for end-stage liver disease (MELD) score, then correlated to the hepatic Doppler parameters and ultrasound (US) findings of hepatic decompensation like ascites and splenomegaly.

Results: By Doppler and US, splenomegaly was evident in $49 \%$ of patients, while ascites was present in $44 \%$ of them. Increased hepatic artery velocity (HAV) was found in $70 \%$ of cases, while 59\% showed reduced portal vein velocity (PW).

There was a statistically significant correlation between HAV and MELD score $(\rho=0.000)$, but no significant correlation with either hepatic artery resistivity index (HARI) $(\rho=0.675)$ or PW $(\rho=0.266)$.

Moreover, HAV had been correlated to splenomegaly $(\rho=0.000)$, whereas HARI $(\rho=0.137)$ and PW $(\rho=0.241)$ did not significantly correlate.

Also, ascites had correlated significantly to MELD score and HAV ( $\rho=0.000)$, but neither HARI $(\rho=0.607)$ nor PW ( $\rho=0.143)$ was significantly correlated.

Our results showed that HAV > $145 \mathrm{~cm} / \mathrm{s}$ could confidently predict a high MELD score with $62.50 \%$ and $97.62 \%$ sensitivity and specificity.

Conclusion: Doppler parameters of hepatic vessels (specifically HAV) in addition to the US findings of hepatic decompensation proved to be a non-invasive and cost-effective imaging tool for severity assessment in cirrhotic patients (scored by MELD); they could be used as additional prognostic parameters for improving the available treatment options and outcomes.
\end{abstract}

Keywords: Hepatic, Artery, Portal, Vein, Doppler, MELD, Cirrhosis

\footnotetext{
* Correspondence: ahmedbaz2012@yahoo.com

${ }^{1}$ Radiology Department, Faculty of Medicine, Cairo University, Cairo, Egypt

Full list of author information is available at the end of the article
}

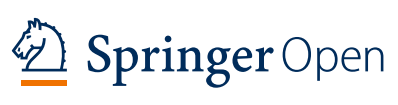

(c) The Author(s). 2020 Open Access This article is licensed under a Creative Commons Attribution 4.0 International License which permits use, sharing, adaptation, distribution and reproduction in any medium or format, as long as you give appropriate credit to the original author(s) and the source, provide a link to the Creative Commons licence, and indicate if changes were made. The images or other third party material in this article are included in the article's Creative Commons licence, unless indicated otherwise in a credit line to the material. If material is not included in the article's Creative Commons licence and your intended use is not permitted by statutory regulation or exceeds the permitted use, you will need to obtain permission directly from the copyright holder. To view a copy of this licence, visit http://creativecommons.org/licenses/by/4.0/. 


\section{Background}

Liver cirrhosis is a worldwide clinical problem that has various infective and metabolic etiologies like hepatitis and alcoholic; the superadded hepatic fibrotic changes and development of regenerative nodules are considered the mainstay culprit of hepatic decompensation and lead to alteration of the hepatic vascular profile that result in portal hypertension [1].

The most commonly utilized (clinical/laboratory) scoring systems for morbidity and mortality prediction in cirrhotic patients are Child-Turcotte-Pugh (CTP) and model for end-stage liver disease (MELD) scores. They could be especially applied for those who would undergo surgeries or interventions. It should be noted that MELD scores of $<10,10$ to 19 , and $>19$ are relevant to CTP classes A, B, and C, respectively [2-4].

MELD score incorporates three available laboratory tests including the serum creatinine, international normalized ratio (INR), and serum bilirubin, and could be calculated using a certain formula $[1,5]$.

However, the MELD score had surpassed CTP in its use, being calculated by more objective parameters (laboratory tests) than do the CTP, moreover, the serum creatinine (one of the MELD parameters) had added value in highlighting the importance of the kidney functions in predicting the mortality risk in end-stage hepatic disease patients [2, 3].

Liver biopsy is an invasive procedure that can be employed for the same purpose but still carrying the bleeding risks and is less favorable by a sector of patients. Fibroscan could be used for assessment of the degree of the hepatic stiffness and detection of cirrhosis [6]; however, studying the vascular hepatic parameters by Doppler Ultrasound and the detection of ultrasound findings of hepatic decompensation in cirrhotic patients with correlation to a reference scoring system like MELD could offer an alternative non-invasive investigation that can be done by the available ultrasound machines in the setting of routine abdominal ultrasound scans.

\section{Aim of work}

To evaluate the Doppler flow parameters of hepatic vessels and the ultrasound findings of hepatic decompensation and investigate any correlation of observed changes with the severity of liver cirrhosis based on the MELD score system.

\section{Methods}

\section{Subjects}

A cross-sectional study was carried out in our institute and enrolled 100 cirrhotic patients (51 males and 49 females) who were recruited for primary screening for hepatocellular carcinoma, during the period from
September 2019 to October 2020, with an age range of 25 to 70 years (mean \pm standard deviation [SD], $56.36 \pm 7.20)$.

\section{Inclusion criteria}

Patients with liver cirrhosis through a combination of the clinical history and typical sonographic findings, including bright coarse hepatic echotexture, nodular liver surface, and widened interlobar fissures.

\section{Exclusion criteria}

The patients who had metastatic disease, hepatocellular carcinoma, or other malignant focal liver masses were excluded from the study.

Written informed consent was obtained from all the patients after explanation of nature and the purpose of our study.

This study was performed after the approval of the local scientific and ethical committee.

All of our patients were subjected to:

1. Full medical history with focusing on risk factors especially for viral hepatitis as blood transfusion, dental procedures, also, the history of symptoms of chronic liver disease (CLD) was checked for.

2. Laboratory investigations, which included the serum creatinine, international normalized ratio (INR), and serum bilirubin. MELD could be calculated according to the following formula $[1,5]$ :

$$
\begin{aligned}
\text { MELD }= & 3.78 \times \ln [\text { serum bilirubin }(\mathrm{mg}=\mathrm{dL})] \\
& +11.2 \times \ln [\mathrm{INR}]+9.57 \\
& \times \ln [\text { serum creatinine }(\mathrm{mg} / \mathrm{dL})]+6.43
\end{aligned}
$$

\section{Doppler and grayscale ultrasound}

a) Grayscale ultrasound: all patients were instructed to fast for at least 6 hours before the ultrasound scan.

The liver was imaged with a standard grayscale using a 3.5 MHz curvilinear transducer using GE Logiq P7 (GE Healthcare, Waukesha, WI). The splenic length was measured in a longitudinal axis during inspiration in a supine position. The presence or absence of ascites was reported.

b) Doppler ultrasound was done through the right lateral intercostal or subcostal approaches to detect 
and interrogate the hepatic vessels. Peak systolic velocity of the hepatic artery velocity (HAV) was measured in centimeters per second $(\mathrm{cm} / \mathrm{s})$ at the hepatic hilum using a corrected Doppler angle of less than or equal to $60^{\circ}$ as an angle of insinuation. The Hepatic Arterial Resistive Index (HARI) was automatically calculated by a dedicated software in the machine or could be manually calculated using the following equation if it was needed:

Resistive index (HARI) $=[$ (peak systolic velocityend-diastolic velocity)/peak systolic velocity] [7]. Portal vein peak velocity (PVV) was measured also in centimeters/second using a corrected Doppler angle of less than or equal to $60^{\circ}$.

\section{4) Statistical methods and data analysis:}

Data was entered and processed using SPSS version 24.0 (SPSS IBM). A comparison between two independent mean groups for parametric data was done using the Student's $t$ test, while descriptive and inferential statistical analyses were carried out as appropriate [8].

Results are expressed in mean $\pm \mathrm{SD}$, and comparisons with $p$ value $<0.05$ were considered as statistically significant [9].

\section{Results}

The Doppler parameters and ultrasound findings were including the following:

\section{Hepatic artery velocity (HAV)}

Our results showed increased HAV in $70 \%$ of the study population; thus, it was considered as an indicator of chronic liver disease. (HAV: mean \pm SD, $91.72 \pm 46.26$ ) (Table 1) (Fig. 1a).

\section{Portal vein velocity (PVV)}

We had noticed a reduction in the PVV, being depicted in $59.0 \%$ of our patients, PVV (mean \pm SD, $19.68 \pm 6.49$ ) (Table 1) (Fig. 1b).

\section{Hepatic artery resistivity index (HARI)}

We had reported a lack of significant correlation between the HARI and the severity of cirrhosis and portal

Table 1 The percentage of hepatic artery and the portal vein velocity thresholds in our patient population

\begin{tabular}{llll}
\hline & & No. & $\%$ \\
\hline Hepatic artery velocity & Decreased $(<30 \mathrm{~cm} / \mathrm{s})$ & 2 & $2.0 \%$ \\
& Normal $(30-60 \mathrm{~cm} / \mathrm{s})$ & 28 & $28.0 \%$ \\
& Increased $(>60 \mathrm{~cm} / \mathrm{s})$ & 70 & $70.0 \%$ \\
Peak velocity of portal vein & Decreased $(<20 \mathrm{~cm} / \mathrm{s})$ & 59 & $59 \%$ \\
& Normal $(20-40 \mathrm{~cm} / \mathrm{s})$ & 34 & $34 \%$ \\
& Increased $(>40 \mathrm{~cm} / \mathrm{s})$ & 7 & $7 \%$ \\
\hline
\end{tabular}

hypertension. HARI (mean $\pm \mathrm{SD}, 0.64 \pm 0.10$ ) (Table 2) (Fig. 1c).

\section{Spleen size correlation}

Out of 100 patients, 49 patients (49\%) had splenomegaly by ultrasound examination. Again, there was a significant correlation between $\mathrm{HAV}$ and splenomegaly $(P=0.000)$, whereas HARI $(\rho=0.137)$ and PVV ( $\rho=0.241)$ did not significantly correlate with splenomegaly (Table 3) (Fig. 2a).

\section{Ascites correlation}

Ascites was present in 44 (out of 100) of our patients (44\%). There was a significant correlation with the MELD score and HAV $(\rho=0.000)$, but HARI $(\rho=$ $0.607)$ and PVV $(\rho=0.143)$ did not show any significant correlation with the presence of ascites (Table 4) (Fig. 2b).

\section{Correlation of Doppler parameters with markers for hepatic decompensation}

The MELD score was available in 100 patients (mean \pm SD, $14.09 \pm 5.49$; MELD score range 6.00-30.00). In our results, there was a statistically significant correlation between HAV and MELD score $(\rho=0.000)$, but no statistically significant correlation with either HARI $(\rho=0.675)$ or PVV ( $\rho=0.266)$. Scatter plots of HAV (Fig. 1a), PVV (Fig. 1b), and HARI (Fig. 1c) as compared with the MELD score were obtained (Table 2).

$(\mathrm{HAV}$ : mean $\pm \mathrm{SD}, 91.72 \pm$ 46.26); HARI (mean \pm SD, $0.64 \pm 0.10) ;$ PVV (mean \pm SD, $19.68 \pm 6.49)$ (Table 2) (Fig. 1a-c).

\section{Diagnostic performance of elevated HAV in predicting hepatic decompensation}

We had suggested that patients with HAV greater than $145 \mathrm{~cm} / \mathrm{s}$ (the cut-off point) can be confidently predicted to have high MELD scores with a sensitivity of $62.50 \%$ and specificity $97.62 \%$ (Table 5)(Fig. 2c, d).

\section{Discussion}

Liver cirrhosis is a well-known clinical entity that has a worldwide distribution with no discernable difference in gender and race, albeit having multiple etiologies; it might have the same longstanding consequences and complications like portal hypertension and hepatic decompensation. It had been agreed that the gold standard reference for the assessment of the degree of cirrhosis is the histological analysis obtained by liver biopsies; however, it should not be the standard care for all cirrhotic patients, being an invasive procedure especially in patients with hemorrhagic tendency [10].

As liver cirrhosis continues, it may alter the hepatic vascular parameters; thus, the Doppler ultrasound scan 


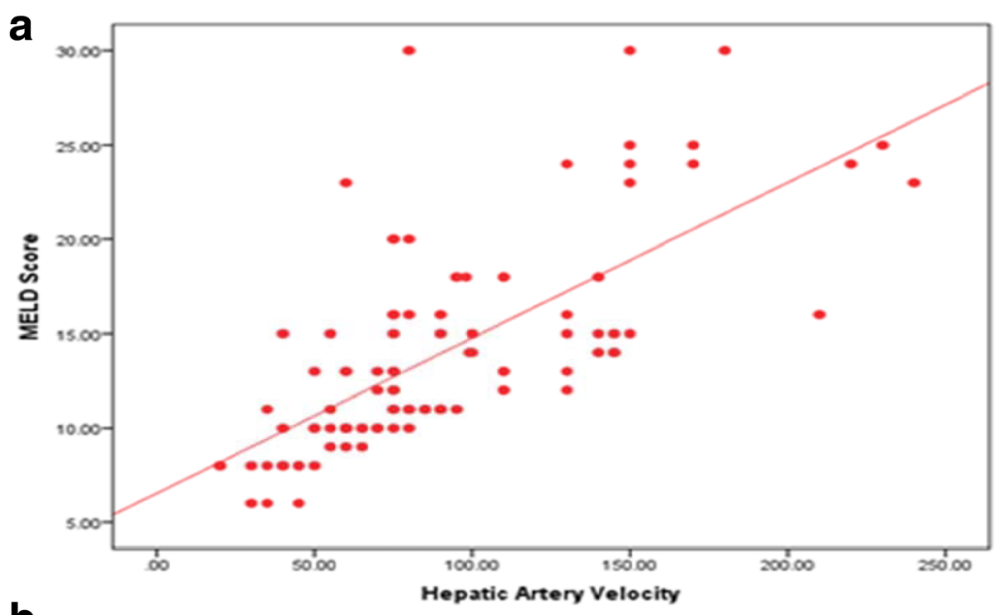

b
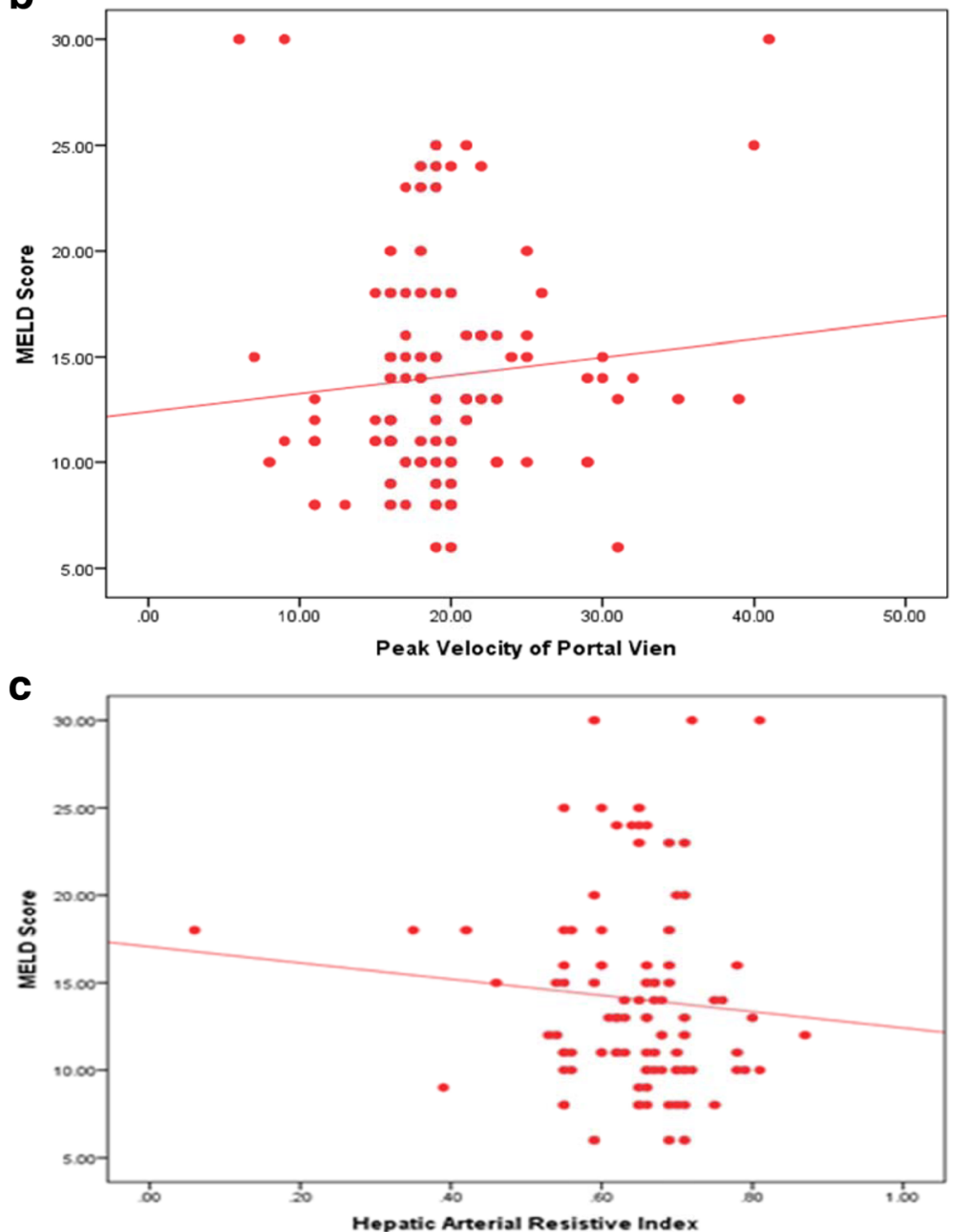

Fig.1 a Scatter plot of MELD score as a function of HAV $(\mathrm{cm} / \mathrm{s})$, the solid line represents the best-fit linear regression. $\mathbf{b}$ Scatter plot of MELD score ( $y$-axis) as a function of PW (cm/s) (x-axis). The solid line represents the best-fit linear regression. c Scatter plot of MELD score ( $y$-axis) as a function of HARI (x-axis). The solid line represents the best-fit linear regression

is warranted as a primary focus for many researchers to be an alternative investigation that could surrogate such invasive procedures [10].
As we mentioned, MELD score system had outperformed the CTP system in patients with chronic liver disease especially those who are candidates for hepatic 
Table 2 Correlation between Doppler ultrasonography findings and MELD score

\begin{tabular}{|c|c|c|c|c|c|c|}
\hline & & MELD $<19$ & MELD > 19 & Test & P- & Sig. \\
\hline & & No. $=84$ & No. $=16$ & & value & \\
\hline \multirow[t]{2}{*}{ Hepatic Artery Velocity } & Median (IQR) & $75(55-100)$ & $150(80-175)$ & -4.092 & 0.000 & $\mathrm{HS}$ \\
\hline & Range & $20-210$ & $60-240$ & & & \\
\hline \multirow[t]{2}{*}{ Hepatic Arterial Resistive Index } & Median (IQR) & $0.66(0.6-0.7)$ & $0.65(0.61-0.71)$ & -0.419 & 0.675 & NS \\
\hline & Range & $0.06-0.87$ & $0.55-0.81$ & & & \\
\hline \multirow[t]{2}{*}{ Peak Velocity of Portal Vein } & Median (IQR) & $19(16-22)$ & $19(18-23.5)$ & -1.113 & 0.266 & NS \\
\hline & Range & $7-39$ & $6-41$ & & & \\
\hline
\end{tabular}

$P>0.05$ non-significant (NS); $P<0.05$ significant (S); $P<0.01$ highly significant (HS) $\bullet$ independent $t$ test, $\cdot$ Mann-Whitney test

transplantation, being more based on objective laboratory biomarkers than do the CTP system; moreover, MELD score does not consider demographic factors like age and race in hepatic transplantation listing; thus, it had been considered as a more favorable scoring system than CTP $[1,3,10]$.

On review of literature, the main objective of this study was to evaluate whether there was a correlation between hepatic Doppler parameters as well as the ultrasound findings of hepatic decompensation with the clinical status in cirrhotic patients as measured by MELD score.

In light of our results, we found a statistically significant correlation between the hepatic artery velocity (HAV) and MELD score (Tables 1 and 2) (Figs. 1a, 3, and 4 ), where $70 \%$ of our cases had showed increased HAV.

This was concordant with Park et al. studies who involved 264 cirrhotic patients and had correlated the Doppler parameters with markers for hepatic decompensation and reported a statistically significant correlation between HAV and MELD score $(\rho=0.0001)[11,12]$; Glisic et al. had also concordant supportive results, but with a relatively smaller sample size (80 patients and 20 controls), and they had reported an increase in HAV of cirrhotic patients with an average $(125.7 \mathrm{~cm} / \mathrm{s})$, compared to their healthy controls $(79.3 \mathrm{~cm} / \mathrm{s})$ [13]; our results had matched theirs, where the cut-off point was $145 \mathrm{~cm} / \mathrm{s}$ (Table 5 and Fig. 2c, d).

A literature-based rationale for such results, where the increased hepatic sinusoidal resistance would, in turn, decrease the portal venous inflow; therefore, it activates the hepatic artery buffer response (HABR; mediated by adenosine washout and angiotensin II system) and increases the hepatic arterial inflow as a compensatory mechanism, to maintain consistent oxygen in the hepatic parenchyma; nevertheless, some studies had documented that HABR is maintained in the setting of cirrhosis $[14,15]$.

Discordance with Azizah et al. study was present in this regard, where they included 56 eligible cirrhotic patients and studied the correlation of hepatic hemodynamics with CTP score, and found that the HAV was not significantly different between the study and the control groups $(\rho=0.06)$. Their possible reason for the contradicting data was that two different US systems were used to scan both control and study groups. No prior checking was carried out by scanning the same patient/s using these two US scanners to look for any significant difference in the measurement of the studied parameters. Also, they were using the CTP score in classifying their patient population, assessing their

Table 3 The spleen size correlation with MELD and the hepatic Doppler parameters

\begin{tabular}{|c|c|c|c|c|c|c|}
\hline & & \multicolumn{2}{|l|}{ Spleen } & \multirow{3}{*}{$\begin{array}{l}\text { Test } \\
\text { value• }\end{array}$} & \multirow{3}{*}{$\begin{array}{l}\mathrm{P} \text { - } \\
\text { value }\end{array}$} & \multirow[t]{3}{*}{ Sig. } \\
\hline & & No & Yes & & & \\
\hline & & No. $=51$ & No. $=49$ & & & \\
\hline \multirow[t]{2}{*}{ MELD Score } & Median (IQR) & $10(9-11)$ & $16(15-23)$ & -8.054 & 0.000 & $\mathrm{HS}$ \\
\hline & Range & $6-15$ & $10-30$ & & & \\
\hline \multirow[t]{2}{*}{ Hepatic Artery Velocity (HAV) } & Median (IQR) & $65(50-80)$ & $110(80-145)$ & -5.754 & 0.000 & $\mathrm{HS}$ \\
\hline & Range & $20-145$ & $35-240$ & & & \\
\hline \multirow[t]{2}{*}{ Hepatic Arterial Resistive Index (HARI) } & Median (IQR) & $0.66(0.61-0.71)$ & $0.65(0.59-0.69)$ & -1.485 & 0.137 & NS \\
\hline & Range & $0.39-0.87$ & $0.06-0.81$ & & & \\
\hline \multirow[t]{2}{*}{ Peak Velocity of Portal Vein (PVV) } & Median (IQR) & $18(16-20)$ & $19(17-23)$ & -2.048 & 0.241 & NS \\
\hline & Range & $7-39$ & $6-41$ & & & \\
\hline
\end{tabular}

$P>0.05$, non-significant (NS), $P<0.05$ significant $(\mathrm{S}), P<0.01$ highly significant $(\mathrm{HS}), \cdot$ independent $t$ test, $\cdot$ Mann-Whitney test 

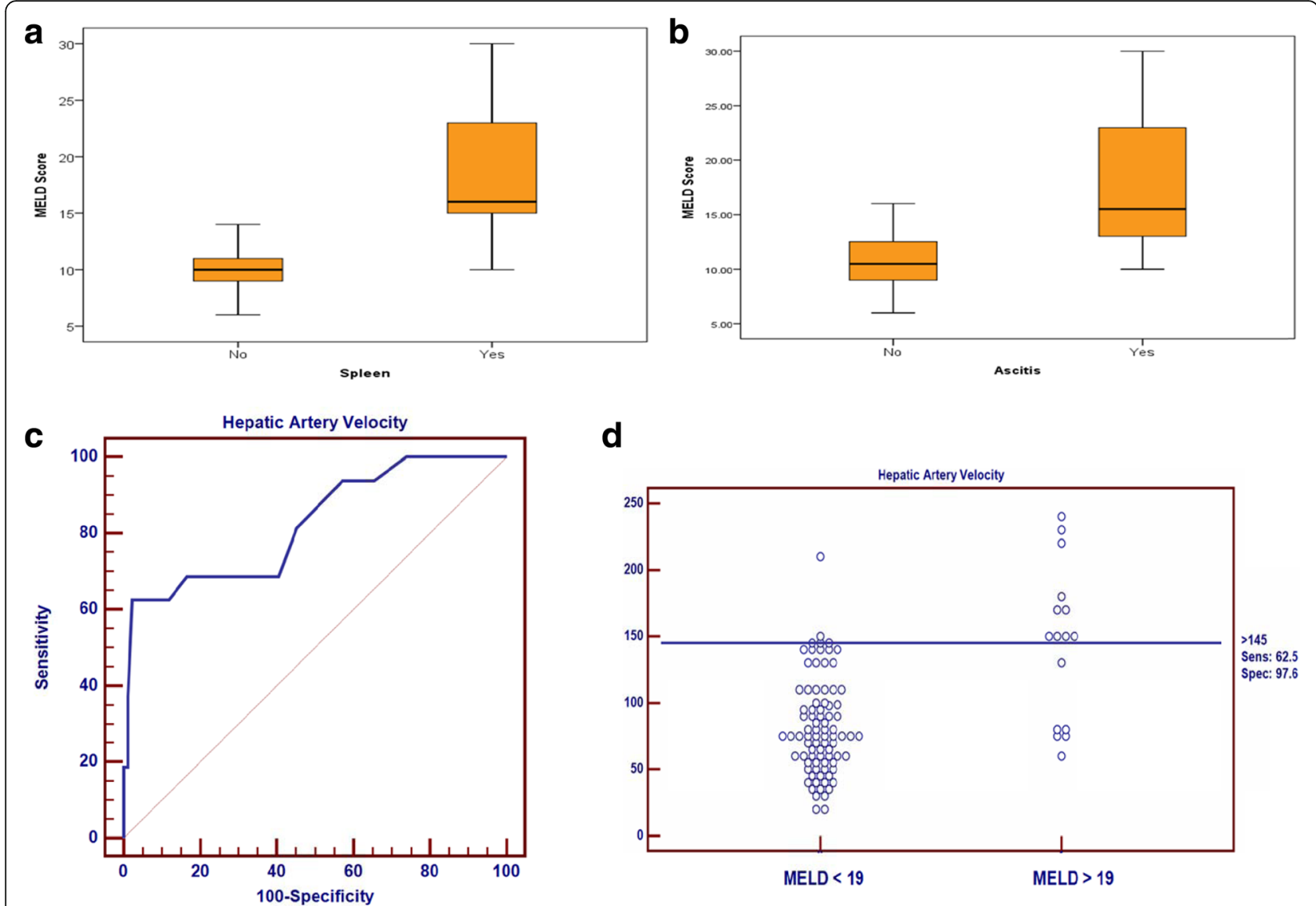

Fig. 2 a Correlation between spleen and MELD by Box plot. b Correlation between ascites and MELD by Box plot. c ROC curve analysis of hepatic artery velocity in prediction of high MELD in cirrhotic patients. Sensitivity $=62.50 \%$, specificity $=97.62 \%$ and cut-off value $>145 \mathrm{~cm} / \mathrm{s}$. $\mathbf{d}$ Scatter plot of MELD score and HAV (cm/s) solid line showing the cut-off point $>145 \mathrm{~cm} / \mathrm{s}$

prognosis, and to predict their mortality. An important deficiency in the CTP score is that it uses 2 very subjective variables in its calculation, namely portosystemic encephalopathy and severity of ascites, again, MELD score calculation is based on more objective parameters [16].
Portal flow velocity was also a studied parameter, although $59 \%$ of our patients showed significantly decreased portal flow velocity $(<20 \mathrm{~cm} / \mathrm{s})$ (mean $\pm \mathrm{SD}$, $19.68 \pm 6.49$ ) (Tables 1 and 2) (Figs. 1b, 3, and 4). We had found that PVV did not significantly correlate with

Table 4 The presence of ascites and correlation with MELD and other Hepatic Doppler parameters

\begin{tabular}{|c|c|c|c|c|c|c|}
\hline & & \multicolumn{2}{|l|}{ Ascites } & \multirow{3}{*}{$\begin{array}{l}\text { Test } \\
\text { value. }\end{array}$} & \multirow{3}{*}{$\begin{array}{l}\text { P- } \\
\text { Value }\end{array}$} & \multirow[t]{3}{*}{ Sig. } \\
\hline & & \multirow{2}{*}{$\begin{array}{l}\text { No } \\
\text { No. }=56\end{array}$} & \multirow{2}{*}{$\begin{array}{l}\text { Yes } \\
\text { No. }=44\end{array}$} & & & \\
\hline & & & & & & \\
\hline \multirow[t]{2}{*}{ MELD Score } & Median (IQR) & $10.5(9-12.5)$ & $15.5(13-23)$ & -6.239 & 0.000 & $\mathrm{HS}$ \\
\hline & Range & $6-25$ & $10-30$ & & & \\
\hline \multirow[t]{2}{*}{ Hepatic Artery Velocity } & Median (IQR) & $70(50-90)$ & $100(75-140)$ & -3.920 & 0.000 & HS \\
\hline & Range & $20-210$ & $40-240$ & & & \\
\hline \multirow[t]{2}{*}{ Hepatic Arterial Resistive Index } & Median (IQR) & $0.66(0.6-0.7)$ & $0.65(0.6-0.7)$ & -0.515 & 0.607 & NS \\
\hline & Range & $0.35-0.87$ & $0.06-0.81$ & & & \\
\hline \multirow[t]{2}{*}{ Peak Velocity of Portal Vein } & Median (IQR) & $19(16.0-20.5)$ & $19(17.5-25.0)$ & -1.829 & 0.067 & NS \\
\hline & Range & $7-31$ & $6-41$ & & & \\
\hline
\end{tabular}


Table 5 The cut-off point, sensitivity and specificity of HAV thresholds to MELD

\begin{tabular}{lllll}
\hline Cut-off point & Sensitivity & Specificity & +PV & -PV \\
\hline$>145 \mathrm{~cm} / \mathrm{s}$ & 62.50 & 97.62 & 83.3 & 93.2 \\
\hline
\end{tabular}

MELD score; this was concordant with many works of literature [16-19] who had documented the same issue; however, this was contradicting the Berzigotti et al. study, where they reported a low flow velocity of $<20$ $\mathrm{cm} / \mathrm{s}$ in addition to a caliber increase in the main portal vein (MPV) were considered diagnostic features of portal hypertension [20]. However, we did not measure the portal vein diameter, and the possible reason for the contradicting data in the PVV was the heterogeneity of the cirrhotic patients included in various studies, also the type of hemodynamic disturbances, for example, the presence of hyperkinetic syndrome or portosystemic collaterals.

Hepatic artery resistivity index (HARI) was additionally investigated in our present study. We found that HARI did not significantly correlate with MELD score, splenomegaly, or ascites (Tables 2, 3, and 4) (Fig. 1c).

This was matching the study done by Iranpour et al., who had observed a specificity and sensitivity of utilizing a hepatic arterial resistive index (RI) cut-off of $>0.77$ to predict cirrhosis were as low as $70 \%$ and $68 \%$, respectively [15], further supportive results had been provided by other studies [7, 11, 13].

This was discordant with the Azizah et al. study, where they found that the increased HARI in the liver cirrhosis was statistically significant and it helped in differentiating cirrhotic participants (where HARI values were above 0.7) from the non-cirrhotic ones (HARI values were less than or equal to 0.7$)$. A possible explanation for the contradicting data is that the RI of the hepatic artery can be changed by certain physiological factors and could be elevated shortly after meal ingestion, where the portal vein flow increases then a corresponding reduction in the arterial flow was induced by vasoconstriction. Nevertheless, the state of vasoconstriction could result in a transient elevation of RI that could last up to few hours [21, 22]; this is why the HARI should be carefully obtained with special consideration for the physiological variations. In our study, we did instruct all patients for 6 $\mathrm{h}$ fasting to avoid this diagnostic bias and we had found no significant correlation.

In the setting of cirrhosis, findings like splenomegaly and ascites could suggest a state of hepatic decompensation, we had depicted a statistically significant correlation between the presence of splenomegaly, MELD score, and HAV; however, this significant correlation was not existing with either PVV or HARI (Table 3)(Figs. 2a, 3, 4). At this extent, we had a concordance
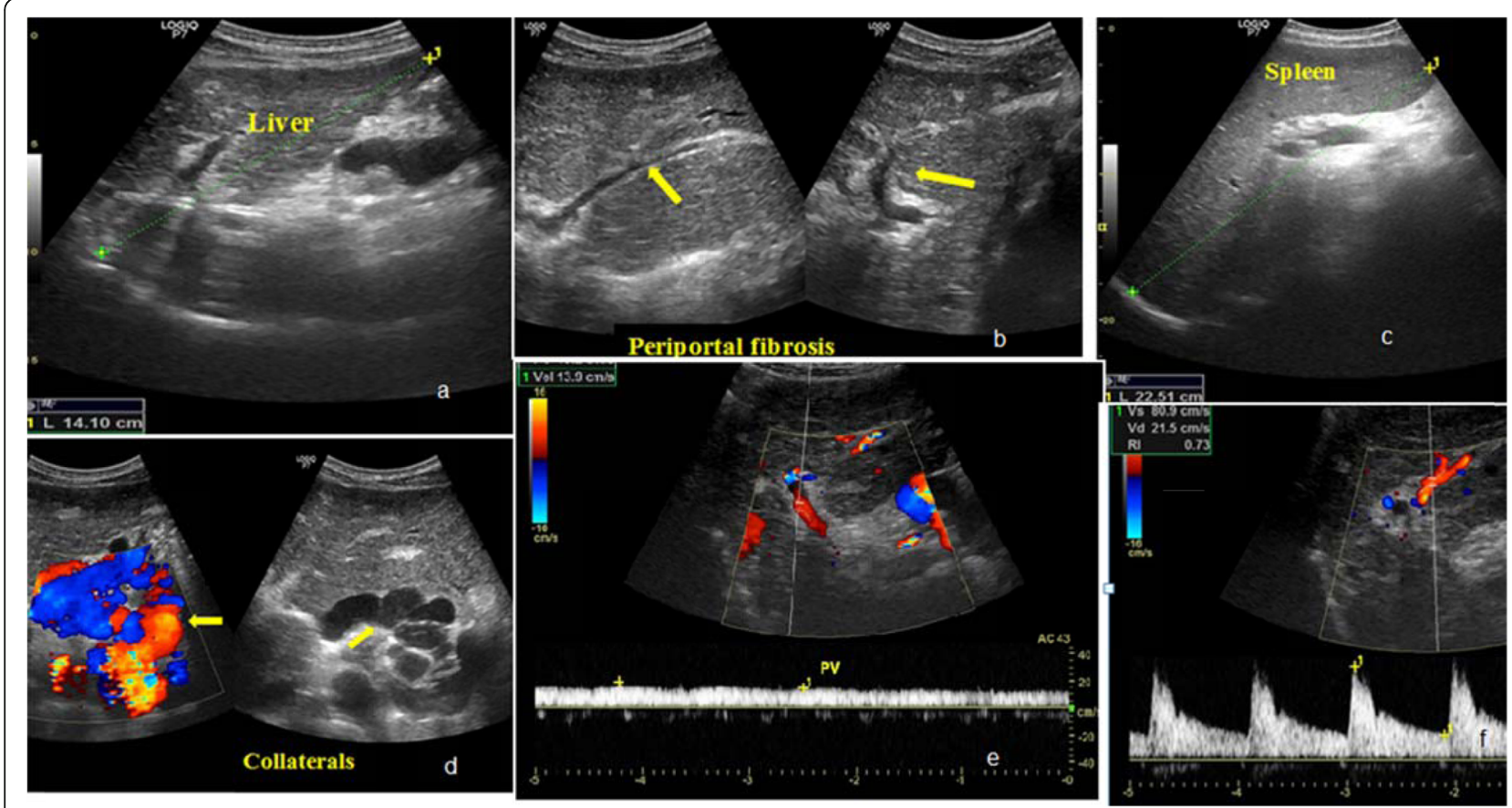

Fig. 3 US images (a-f). a, b Grayscale images showing a cirrhotic liver with uneven surface and periportal fibrosis (yellow arrows). c Grayscale image showing splenomegaly (length $=22 \mathrm{~cm}$ ). $\mathbf{d}$ Color Doppler image showing a cluster of gastro-esophageal porto systemic collaterals (yellow arrows). e Spectral Doppler image tracing the PW that was $13.9 \mathrm{~cm} / \mathrm{s}$ (reduced) [Normal = $20-40 \mathrm{~cm} / \mathrm{s}$ ]. f Spectral Doppler image tracing the $\mathrm{HAV}$ and HARI, where the HAV is elevated $=80.9 \mathrm{~cm} / \mathrm{s}[$ Normal $=30-60 \mathrm{~cm} / \mathrm{s}]$, it also shows abnormal high $\mathrm{HARI}=0.73[\mathrm{Normal}=0.60-0.68]$ 


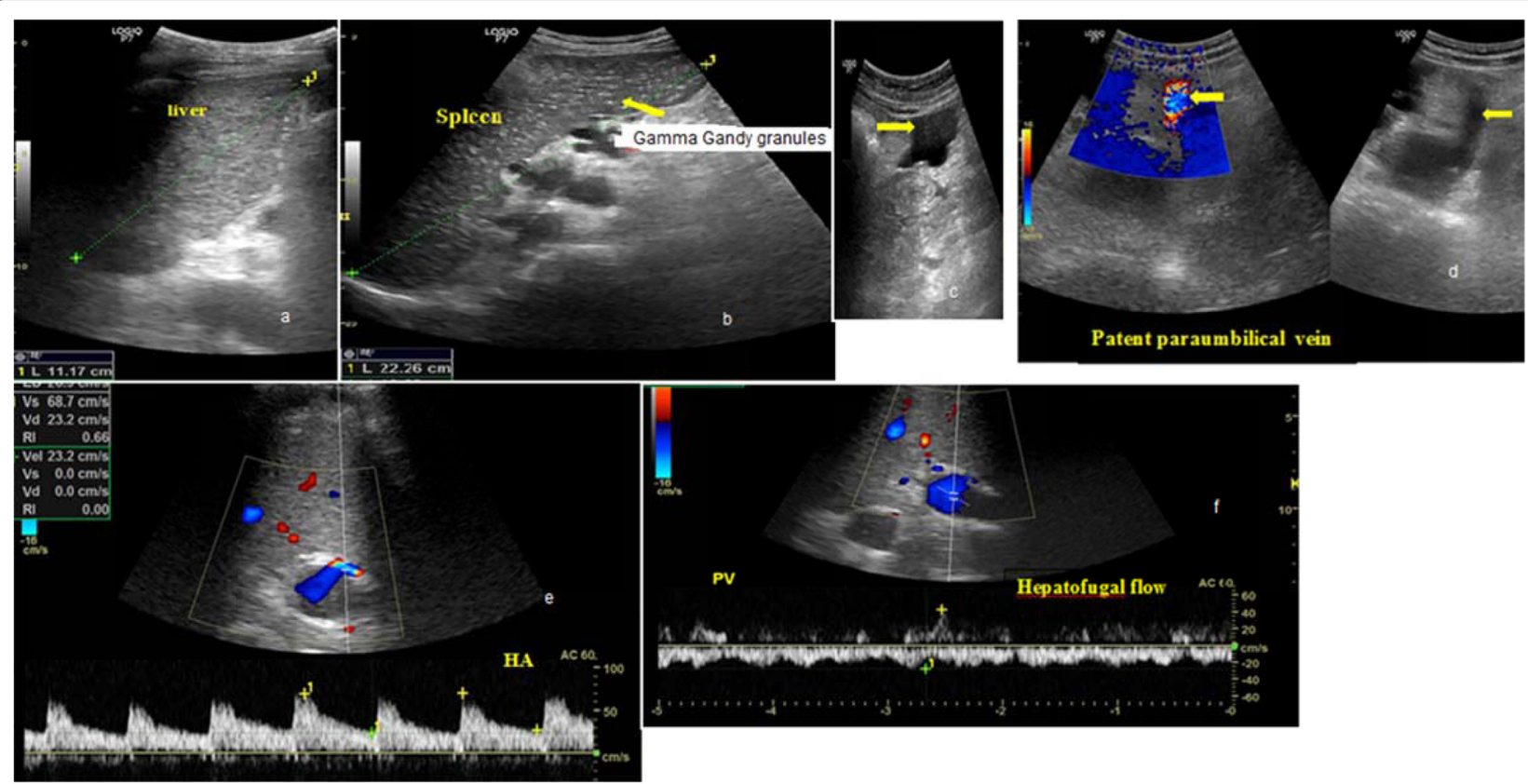

Fig. 4 US images (a-f). a-c Grayscale images showing a cirrhotic liver (a) splenomegaly (b) (length $=22 \mathrm{~cm}$ ) with a myriad of echogenic nonshadowing foci representing Gamma-Gandy nodules (yellow arrow)(an association with longstanding portal hypertension). c Mild amount of free ascites was noted (yellow arrow). $\mathbf{d}$ Color Doppler and grayscale images showing recanalized para-umbilical vein (yellow arrows). e Spectral Doppler image tracing the main portal vein at the porta hepatis showing a reversed hepatofugal (away from the liver) flow. $\mathbf{f}$ Spectral Doppler image tracing the HAV and HARI, where the HAV is elevated $=68.7 \mathrm{~cm} / \mathrm{s}$, and HARI was normal $=0.66$

with many studies [11, 23-25], but on the other hand, it was discordant with the Yadav et al. study [26], who found (out of 112 patients) no significant correlation between MELD score and spleen size in cirrhosis.

Kashani et al. had explained this discordance, where the size of the spleen could vary between cirrhotic patients according to their primary disease etiologies, with hepatitis $\mathrm{C}$ virus (HCV) infected and non-alcoholic hepatitis patients were showing significantly larger organ dimensions as compared to those with alcoholic hepatitis [27], almost all of our patients lie in the category of post-infection (hepatitis) cirrhosis, where the splenomegaly was accordingly a harmonious feature. It worth mentioning, that some authors had suggested that splenomegaly is associated with a poor prognosis in cirrhotic patients and could be used as an index for the non-invasive assessment of esophageal varices and bleeding risks during radioactive or acoustic examinations $[28,29]$.

Ascites is another important complication of cirrhosis and is usually associated with poor prognosis (1- and 5 year moralities are $15 \%$ and $44 \%$, respectively), on follow-up [30].

The current study showed a statistically significant correlation between the presence of ascites and the MELD score as well as HAV. But on the other side, the correlation with both PVV and HARI (Tables 2 and 4)
(Figs. 2b and 4) was statistically insignificant; this was in line with Azizah et al., Park et al., and Somsouk et al. studies [11, 16, 30].

Yet, Popov et al. had evaluated and compared the changes of hepatic and renal Doppler parameters in cirrhotic patients, and found no significant differences between hepatic Doppler parameters and the presence or absence of ascites [31]. Our explanation for this mismatch was attributed to the different stages of hepatic cirrhosis in their patients who could have a previous taping or had received treatments for ascites. But most of our ascetic patients had a decompensated end-stage liver disease and gave no history of previous surgical intervention or the transjugular intrahepatic portosystemic shunt (TIPS) to treat their ascites.

Although providing potentially useful data, we had some limitations in this work including the following:

First, in this study, we did not correlate the degree of cirrhosis or confirm the presence of cirrhosis on histologic bases. Although we agree that histology is considered a reference standard, it was not the standard protocol to have a hepatic biopsy in all cirrhotic patients. Second, we did not include normal controls in our study, as we consider that the MELD score has only been used in cirrhotic patients and is not validated to be used in normal individuals. Moreover, normal individuals do not have regularly checked laboratory tests for MELD to 
provide a fair comparison. Third, is that our study sample was from a single center. Finally, the study duration was relatively short (about 12 months) with a relatively small sample size of recruited patients; therefore, statistical analysis may not be so robust.

\section{Conclusion}

Doppler US of hepatic vessels (specifically the HAV) in addition to the ultrasound findings of hepatic decompensation like splenomegaly and ascites had proved to be a non-invasive and cost-effective imaging tool for assessment of the severity in chronic liver disease patients (as scored by MELD); they could be used as additional prognostic parameters for improving the available treatment options and outcomes.

\section{Acknowledgements}

Not applicable

\begin{abstract}
Authors' contributions
$A A B$ : the corresponding author had contributed by supervising the ultrasound examinations and in the final editing and submission of the manuscript. RMM had done the ultrasound examinations for the patients and shared in the manuscript editing and reference collection. KHE: had introduced the idea of the current study and helped in the image selection and revised the final version of the submitted manuscript. All author(s) read and approved the final manuscript.
\end{abstract}

\section{Funding}

All authors had no fund for this research and had no competing interests.

\section{Availability of data and materials}

All data are available on a software system owned by each of the authors and the corresponding author has the authority to respond if there is any query.

\section{Ethics approval and consent to participate}

The protocol was reviewed and approved by the local ethics committee of the radiology department, Kasr Alainy Hospital, Cairo University. The reference number: Code MS-63_2019, date of approval: 28 September 2019. All patients had given their written consents to participate in this work.

\section{Consent for publication}

All patients had given their written consents for publication of this work.

\section{Competing interests}

All authors declare that they have no competing interests.

\section{Author details}

${ }^{1}$ Radiology Department, Faculty of Medicine, Cairo University, Cairo, Egypt.

${ }^{2}$ Faculty of Medicine, Cairo University, Cairo, Egypt.

Received: 30 September 2020 Accepted: 27 October 2020

Published online: 14 November 2020

\section{References}

1. Aranzana EM, Coppini AZ, Ferreira FG et al (2015) Model for End-Stage Liver Disease, model for liver transplantation survival and donor risk index as predictive models of survival after liver transplantation in 1,006 patients. Clinics 70(6):413-418

2. Abbas N, Makker J, Abbas H et al (2017) Perioperative care of patients with liver cirrhosis: A review. Health Serv Insights. 10:117863291769127

3. Merion RM, Wolfe RA, Dykstra DM et al (2003) Longitudinal assessment of mortality risk among candidates for liver transplantation. Liver transplantation. 9(1):12-18
4. Wiesner R, Edwards E, Freeman R et al (2003) Model for end-stage liver disease (MELD) and allocation of donor livers. Gastroenterology. 124(1): 91-96

5. Kamath PS, Kim WR (2007) The model for end-stage liver disease (MELD). Hepatology 45:797-805

6. Elzawawy MS, Hassanein SA, El Nomrosy RM (2018) The role of fibroscan in the assessment of liver cirrhosis in patients with chronic liver disease. Menoufia Med J 31:520-524

7. Lim AK, Patel N, Eckersley RJ et al (2005) Can Doppler sonography grade the severity of hepatitis C-related liver disease? Am J Roentgenol 184:1848-1853

8. Chan YH (2003) Biostatistics 102: quantitative data--parametric \& nonparametric tests. Singapore Med J 44(8):391-396

9. Wasserstein RL, Lazar NA (2016) The ASA statement on P-value: context, process, and purpose. Am Statistic. 70:129-133

10. Topal NB, Sarkut P, Dündar HZ et al (2016) The correlation between Doppler US measurement of hepatic arterial flow and the MELD score in patients with chronic liver disease. Eur Rev Med Pharmacol Sci 20(2):291

11. Park HS, Desser TS, Jeffrey RB et al (2016) Doppler ultrasound in liver cirrhosis: Correlation of hepatic artery and portal vein measurements with model for end-stage liver disease score. J Ultrasound Med 36: 725-730

12. Park HS, Desser TS, Jeffrey RB et al (2017) Doppler ultrasound in liver cirrhosis: Correlation of hepatic artery and portal vein measurements with model for end-stage liver disease score. J Ultrasound Med 36: 725-730

13. Glisic TM, Perisic MD, Dimitrijevic S et al (2014) Doppler assessment of rrelation with ammonia plasma levels and MELD score. J Clin Ultrasound. 42:264-269

14. Iranpour P, Lall C, Houshyar R et al (2016) Altered Doppler flow patterns in cirrhosis patients: an overview. Ultrasonography. 35(1):3-12

15. Ferreira, N., Costa, Marques, Â. et al., (2016): Doppler Ultrasound Evaluation in Portal Hypertension.

16. Afif A, JasonChang, YanWanget al. (2017) A sonographic Doppler study of the hepatic vein, portal vein and hepatic artery in liver cirrhosis: correlation of hepatic hemodynamics with clinical Child-Pugh score in Singapore. Ultrasound 25(4):213-221

17. Abdel-RazikaA MN, Elhelaly $R$ et al (2015) De-novo portal vein thrombosis in liver cirrhosis: risk factors and correlation with the Model for End-stage Liver Disease scoring system. Eur J Gastroenterol Hepatol 27:585-592

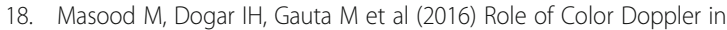
Evaluation of Hemodynamic Changes in Chronic Hepatitis C Infection. Ann Intern Med 22(2):135-138

19. Achim CA, Bordei P, Dumitru Eet al. (2016) The role of ultrasonography in the evaluation of portal hemodynamics in healthy adults and pathologic conditions. ARS Medica Tomitana 22(2):128-134

20. Berzigotti A, Seijo S, Reverter E et al (2013) Assessing portal hypertension in liver diseases. Expert Rev Gastroenterol Hepatol 7:141-155

21. Lafortune M, Dauzat M, Pomier G et al (1993) Hepatic artery, the effect of meal persons, and transplant recipient. Radiology 187:391-394

22. Joynt LK, Platt JF, Rubin JM et al (1995) Hepatic artery resistance before and after a standard meal in subjects with diseased and healthy livers. Radiology 196(2):489-492

23. O'Donohue J, Ng C, Catnach S et al (2004) Diagnostic value of Doppler assessment of the hepatic and portal vessels and ultrasound of the spleen in liver disease. Eur J Gastroenterol Hepatol 16:149-155

24. Nuray H, Esra O, Ayse E et al (2013) Are caudate-right lobe ratio and splenic size correlated with the MELD score in cirrhotic patients? Clin Imaging 37 : $526-529$

25. Schneider AR, Teuber G, Kriener S et al (2005) Noninvasive assessment of liver steatosis, fibrosis and inflammation in chronic hepatitis $C$ virus infection. Liver Int 25(6):1150-1155

26. Yadav M, Devdas K, Mathew J et al (2018) Correlation between spleen size, platelet count, and MELD score in cirrhosis. J Clin Exp Hepatol 8:S61

27. Kashani A, Salehi B, Anghesom D et al (2015) Spleen size in cirrhosis of different etiologies. J Ultrasound Med. 34(2):233-238

28. Mashrafi A, Fawwad Z, Tahmina B et al (2015) Correlation Between Spleen Size, Thrombocytopenia and Meld Score in Chronic Liver Disease and Their Relation with Hospital Readmission. Blood 126(23):5567 
29. Colecchia A, Montrone L, Scaioli E et al (2012) Measurement of spleen stiffness to evaluate portal hypertension and the presence of esophageal varices in patients with HCV-related cirrhosis. Gastroenterology. 143(3):646-654

30. Somsouk MA, Kornfield R, Vittinghoff E et al (2011) Moderate ascites identifies patients with low MELD awaiting liver transplantation with high mortality risk. Liver Transpl 17(2):129-136

31. Popov D, Krasteva R, Ivanova R et al (2012) Doppler parameters of hepatic and renal hemodynamics in patients with liver cirrhosis. Int J Nephrol 2012: 961654

\section{Publisher's Note}

Springer Nature remains neutral with regard to jurisdictional claims in published maps and institutional affiliations.

\section{Submit your manuscript to a SpringerOpen ${ }^{\mathcal{O}}$ journal and benefit from:}

- Convenient online submission

- Rigorous peer review

- Open access: articles freely available online

High visibility within the field

- Retaining the copyright to your article

Submit your next manuscript at $\boldsymbol{\nabla}$ springeropen.com 